\title{
Preparation and Characterization of Co Ferrite Thin Films on MgO (100) Substrates by Metal Organic Decomposition
}

\author{
Minami Ninomiya, Michimasa Sasaki, Takao Tsurui, \\ Kenji Shinozaki, Takayuki Komatsu and Takayuki Ishibashi* \\ Department of Materials Science and Technology, Nagaoka University of Technology, \\ 1603 Kamitomioka, Nagaoka, Niigata 940-2188, Japan
}

(Received February 13, 2015; accepted May 21, 2015)

Key words: Co ferrite, metal organic decomposition, thin film

Co ferrite thin films were prepared on $\mathrm{MgO}$ (100) substrates by metal organic decomposition (MOD). Crystalline structure, surface morphology, magnetic properties, and composition were investigated. The X-ray diffraction (XRD) pattern of the Co ferrite thin films indicates that $\mathrm{CoFe}_{2} \mathrm{O}_{4}$ was grown. A transmission electron microscopy measurement reveals that the Co ferrite thin film prepared by MOD has a layered structure. Magnetic properties were measured to study the magnetic anisotropy of the Co ferrite films.

\section{Introduction}

Magnetic thin films exhibiting a large perpendicular magnetic anisotropy (PMA) of over $10^{7} \mathrm{erg} / \mathrm{cm}^{3}$ are now in great demand for potential applications in ultrahigh-density magnetic recording media $\left(>1 \mathrm{Tbit} / \mathrm{inch}^{2}\right) .{ }^{(1)} \mathrm{FePt}, \mathrm{CoPt}, \mathrm{SmCo}$, and $\mathrm{NdFeB}$ are known as materials having large magnetic anisotropy constants $\left(K_{\mathrm{u}}\right)$. For example, the $K_{\mathrm{u}}$ values of FePt and CoPt are $7 \times 10^{7} \mathrm{erg} / \mathrm{cm}^{3}$ and $2.8 \times 10^{7}$, respectively. ${ }^{(2)}$ However, these materials include rare-earth elements and/or noble metals. In recent years, materials for next-generation high-density magnetic recording media that do not contain precious elements have been required.

Co ferrite is a classical ferrimagnetic material. Figure 1 shows the structure of Co ferrite. This material has an inverse spinel structure in which eight tetrahedral cation A-sites are occupied by eight $\mathrm{Fe}^{3+}$ ions, while the sixteen octahedral cation B-sites are randomly occupied with $\mathrm{Fe}^{2+}$ and $\mathrm{Co}^{2+}$ ions. Recently, it has been reported that epitaxial $\mathrm{Co}_{x} \mathrm{Fe}_{3-x} \mathrm{O}_{4}(x=0.75$ and 1.0) thin films on $\mathrm{MgO}$ (100) substrates prepared by reactive RF magnetron sputtering showed a large PMA with $K_{\mathrm{u}}$ values estimated at $9.0 \times 10^{6}$ and 9.7 $\times 10^{6} \mathrm{erg} / \mathrm{cm}^{3}$ for $x=0.75$ and 1.0 , respectively. ${ }^{(3)}$

${ }^{*}$ Corresponding author: e-mail: t_bashi@mst.nagaokaut.ac.jp 


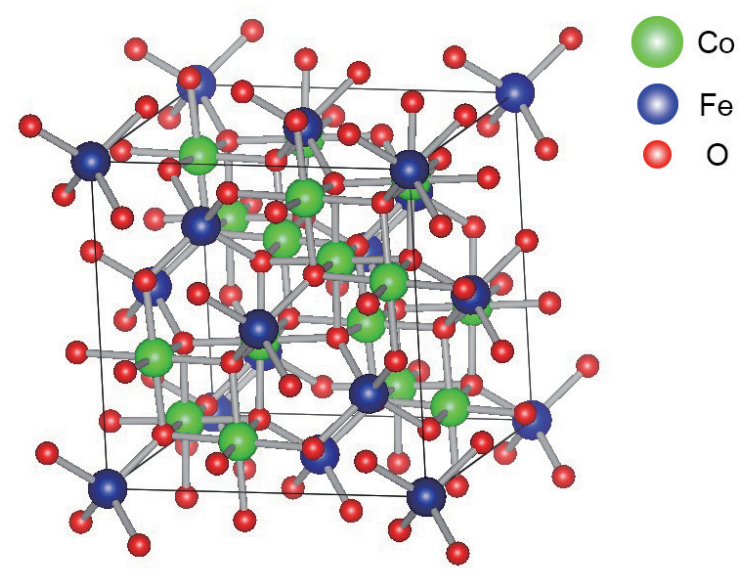

Fig. 1. (Color online) Crystal structure of Co ferrite.

In this study, we used metal organic decomposition (MOD) to prepare the materials; MOD is a simple method with several advantages, such as the ability to fabricate a large area and to control chemical compositions. Therefore, MOD is promising for exploring new materials. However, there have been no reports yet of Co ferrite films prepared by MOD. In this paper, we report on Co ferrite thin films prepared on $\mathrm{MgO}$ (100) substrates by MOD.

\section{Experiments}

Co ferrite thin films were prepared by MOD. A MOD solution (Kojundo Chemical $\mathrm{Lab}$ Co., Ltd.) with the ratio of $\mathrm{Co}: \mathrm{Fe}=1: 2$ and the total concentration of metalorganic materials of $4 \%$ was spin-coated on a $\mathrm{MgO}(100)$ substrate at $3000 \mathrm{rpm}$ for $30 \mathrm{~s}$, followed by drying at $100{ }^{\circ} \mathrm{C}$ for $10 \mathrm{~min}$ on a hot plate. To decompose organic materials and to obtain precursor films, samples were preannealed at $450{ }^{\circ} \mathrm{C}$ for $10 \mathrm{~min}$. These processes, i.e. spin-coating, drying, and preannealing, were repeated five times. Finally, the samples were annealed for crystallization in an electric furnace for $1 \mathrm{~h}$ at 550,700, or $850{ }^{\circ} \mathrm{C}$. All thermal treatments were performed in air.

The crystalline structures of these films were analyzed by X-ray diffraction (XRD) (SmartLab, Rigaku). Surface morphology was observed by atomic force microscopy (AFM) (Nanocute, Hitachi High-Tech Science Co.). A vibrating sample magnetometer (VSM) was used to measure magnetization at room temperature. The composition and thickness were measured by transmission electron microscopy (TEM) (JEM-2100, JEOL) combined with energy dispersive X-ray (EDX) spectroscopy. 


\section{Ressults and Discussion}

Figure 2 shows XRD ( $\omega-2 \theta$ scan) patterns of Co ferrite thin films on $\mathrm{MgO}(100)$ substrates. The 400 diffraction peaks of $\mathrm{CoFe}_{2} \mathrm{O}_{4}$ thin films with relatively strong intensities are found to the right of the 200 diffraction peaks of the $\mathrm{MgO}$ substrate observed at $42.8^{\circ}$, indicating that the $\mathrm{CoFe}_{2} \mathrm{O}_{4}$ thin films grow with (100) orientation. The 400 peaks sharpen as the annealing temperature is increased. The lattice constants along [100] of the Co ferrite thin films annealed at 700 and $850{ }^{\circ} \mathrm{C}$ were estimated to be 8.344 and $8.366 \AA$, respectively, from the angles of the 400 diffraction peaks, both of which are smaller than that of the $\mathrm{CoFe}_{2} \mathrm{O}_{4}$ bulk at $8.381 \AA .{ }^{(4)}$ This result indicates that the films have a distortion caused by tensile strain in the in-plane direction due to lattice mismatching between $\mathrm{CoFe}_{2} \mathrm{O}_{4}$ and $\mathrm{MgO}$, resulting in the shrinkage of the lattice constant in the out-of-plane direction. The effective out-of-plane strains are -0.441 and $-0.179 \%$ for the samples annealed at 700 and $850{ }^{\circ} \mathrm{C}$, respectively. The induced strain could give rise to significant effects in the magnetocrystalline anisotropy. On the other hand, the 311 diffraction peaks of $\mathrm{CoFe}_{2} \mathrm{O}_{4}$ are observed at $35.4^{\circ}$ in all samples, suggesting the mixture of epitaxially grown $\mathrm{CoFe}_{2} \mathrm{O}_{4}$ film and polycrystalline film as described later.

Figure 3 shows VSM hysteresis curves of the Co ferrite thin film annealed at $700{ }^{\circ} \mathrm{C}$. Coercive forces are approximately $1 \mathrm{kOe}$ in both in-plane and out-of-plane directions. Although the easy axes of magnetization are in the in-plane direction, the hysteresis curves for both directions are almost the same if the demagnetization field is taken into account as shown in Fig. 3(b). This result indicates that there is no specific direction of magnetic anisotropy. We also obtained similar results in the other films (not shown).

Figure 4 shows AFM images of the Co ferrite thin films. We found that the surface morphology of all the samples has a granular structure, and the grain size becomes larger as the temperature is increased. The average grain sizes for the thin films annealed at 550,700 , and $850{ }^{\circ} \mathrm{C}$ are approximately 25,50 , and $100 \mathrm{~nm}$, and the averages roughness

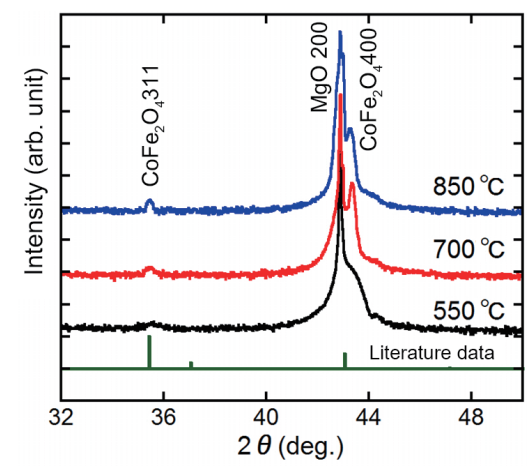

Fig. 2. (Color online) XRD patterns of Co ferrite thin films. 


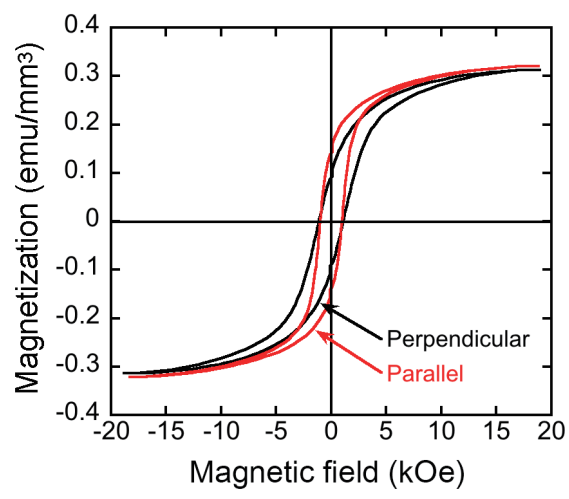

(a)

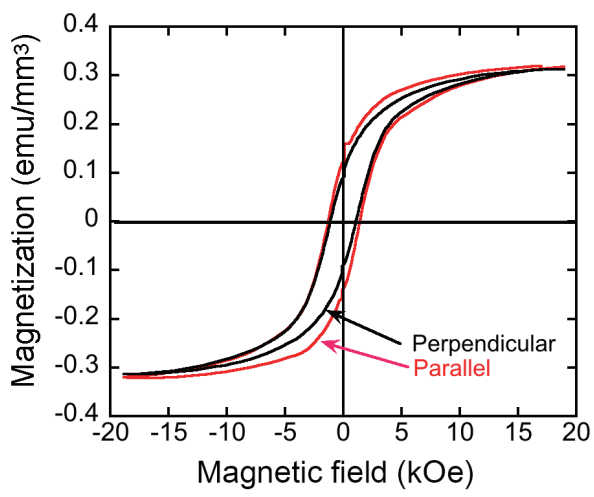

(b)

Fig. 3. (Color online) VSM hysteresis curves of Co ferrite thin film annealed at $700{ }^{\circ} \mathrm{C}$ : (a) measured data and (b) including consideration of demagnetization data.

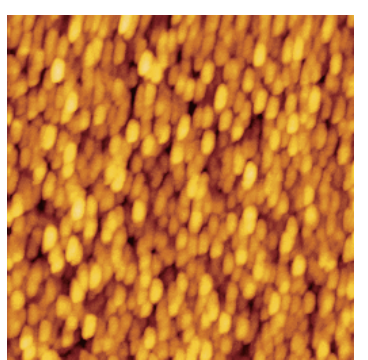

(a)

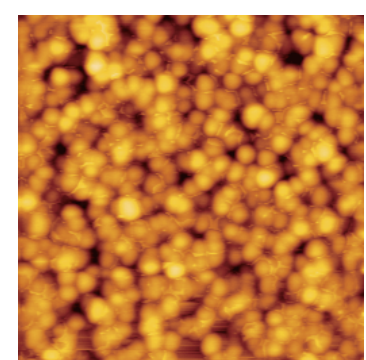

(b)

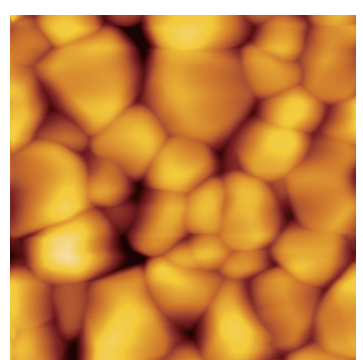

(c)

Fig. 4. (Color online) AFM images of Co ferrite thin films annealed at (a) 550, (b) 700, and (c) $850^{\circ} \mathrm{C}$.

$(R a)$ values for thin films annealed at 550,700 , and $850{ }^{\circ} \mathrm{C}$ are $0.42,1.29$, and $7.37 \mathrm{~nm}$, respectively.

Figure 5 shows high-resolution TEM images of the Co ferrite thin film annealed at $700{ }^{\circ} \mathrm{C}$. The thickness of the Co ferrite thin film is $393 \mathrm{~nm}$. We found that it has a layer structure corresponding to the number of spin-coatings, for which there is a clear contrast consisting of black and white areas. Magnified TEM images revealed that the first layer was grown epitaxially on the $\mathrm{MgO}$ substrate, and the other layers, from the 2nd to 5th layers, have a polycrystalline structure. In addition, we found that the white area is a void structure.

Figure 6 shows electron diffraction patterns of a Co ferrite thin film annealed at $700{ }^{\circ} \mathrm{C}$. The diffraction pattern of the first layer in Fig. 6(b) shows a periodic pattern corresponding to the Co ferrite, and it has the same orientation as the $\mathrm{MgO}$ substrate, 

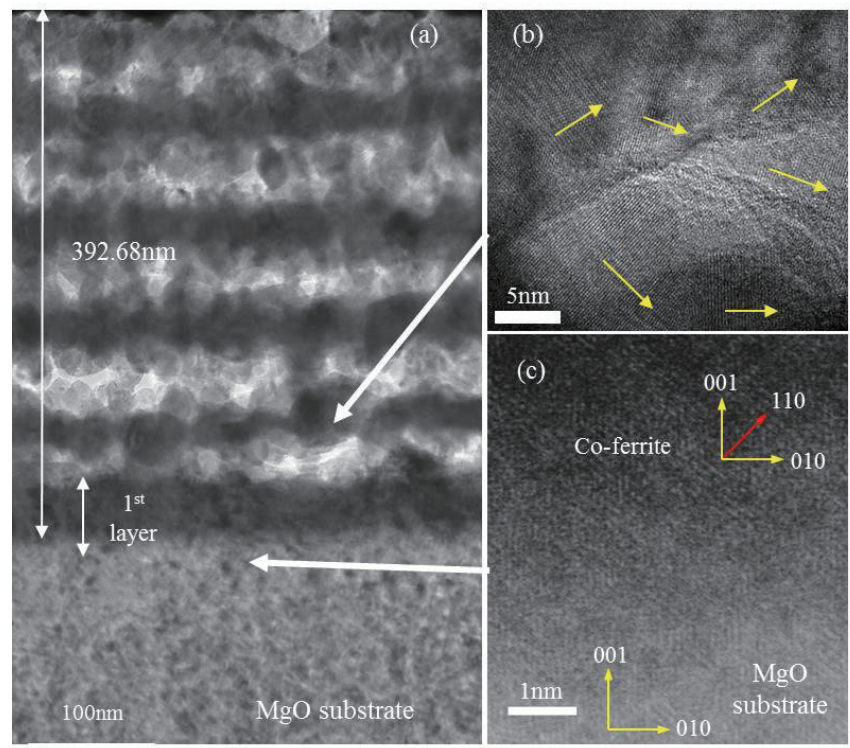

Fig. 5. (Color online) (a) High-resolution TEM images of Co ferrite thin film annealed at $700{ }^{\circ} \mathrm{C}$, (b) the second layer, and (c) around the interface between the film and the MgO substrate.

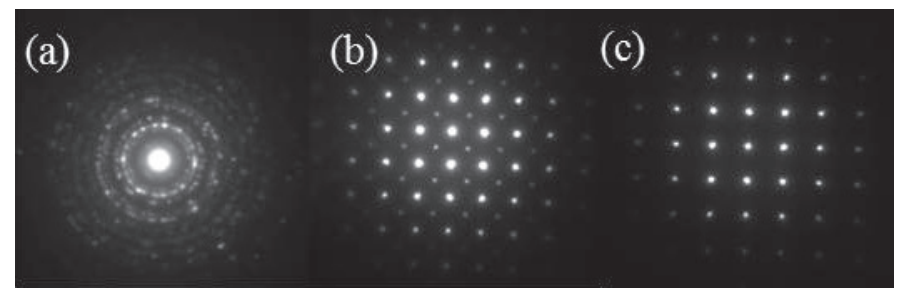

Fig. 6. Electron diffraction patterns of Co ferrite thin film annealed at $700{ }^{\circ} \mathrm{C}$ : (a) the middle of the layer, (b) around the interface between the films and $\mathrm{MgO}$ substrate, and (c) $\mathrm{MgO}$ substrate. (a) and (b) are measured at the same positions shown in Figs. 5(b) and 5(c), respectively.

indicating epitaxial growth of Co ferrite. In contrast, the second layer shows a ring pattern, indicating a polycrystalline structure.

Table 1 shows a summary of the chemical compositions of each layer in the Co ferrite thin film annealed at $700{ }^{\circ} \mathrm{C}$ determined by EDX spectroscopy. We found that the composition of the second to the fourth layers, including the bright contrast area, is almost the same as that of the MOD solution. However, the ratio of Co to Fe is 1:3 for the first layer, and the composition of the fifth layer is higher in Co than that of the MOD solution, suggesting that the excess Co atoms moved to the surface. 
Table 1

Compositions of $\mathrm{Co}$ and $\mathrm{Fe}$ of the Co ferrite thin film annealed at $700{ }^{\circ} \mathrm{C}$ measured by EDX. The layer number corresponds to the order of spin coating.

\begin{tabular}{lcc}
\hline \multirow{2}{*}{ Layer number } & \multicolumn{2}{c}{ Composition ratio (atom \%) } \\
\cline { 2 - 3 } & Co & Fe \\
\hline 5 & 36 & 64 \\
4 & 34 & 66 \\
3 & 34 & 66 \\
2 & 34 & 66 \\
1 & 25 & 75 \\
\hline
\end{tabular}

We suggest that the change in composition of the first layer is associated with the lattice matching with the $\mathrm{MgO}$ substrate. The lattice constant of $\mathrm{Co}_{0.75} \mathrm{Fe}_{2.25} \mathrm{O}_{4}$ is expected to be $8.385 \AA$ using Vegard's law. This value is larger than that of $\mathrm{CoFe}_{2} \mathrm{O}_{4}(a$ $=8.34 \AA$ ) and is closer to double the lattice constant of the $\mathrm{MgO}$ substrate $(a=4.21 \AA$ ). Therefore, $\mathrm{Co}_{0.75} \mathrm{Fe}_{2.25} \mathrm{O}_{4}$ was grown epitaxally on the $\mathrm{MgO}$ substrate so that the lattice mismatching became smaller. On the other hand, polycrystalline $\mathrm{CoFe}_{2} \mathrm{O}_{4}$ films were grown in the other layers because there was no restriction on crystal growth.

Finally, we discuss the bright contrast area observed in the area between layers in Fig. 5(a). We found that the intensity of the EDX spectra is weaker than that of the dark area. The density of the bright area must be lower than that of the dark area, since the difference in contrast is related to the film density. Therefore, we suggest that the bright areas include voids. Considering the MOD process, the voids may be created during the decomposition of organic materials. This means that the preannealing process for the decomposition of organic components is unsufficient. If organic components remain in the film after the preannealing process, gasses such as $\mathrm{CO}_{2}$ and $\mathrm{H}_{2} \mathrm{O}$ are produced during the annealing process for crystallization at high temperatures, causing voids in the films.

\section{Conclusions}

We studied Co ferrite thin films grown on $\mathrm{MgO}$ (100) substrates by MOD. The lattice constants along [100] of the Co ferrite thin films annealed at 700 and $850{ }^{\circ} \mathrm{C}$ were estimated to be 8.344 and $8.366 \AA$, respectively, indicating that the effective out-of-plane strains were -0.441 and $-0.179 \%$. There was no clear magnetic anisotropy for any of the Co ferrite thin films prepared in this study. TEM images revealed that $\mathrm{Co}_{0.75} \mathrm{Fe}_{2.25} \mathrm{O}_{4}$ was grown epitaxially on the $\mathrm{MgO}$ substrate for the first layer and polycrystalline $\mathrm{CoFe}_{2} \mathrm{O}_{4}$ was grown in the other layers.

\section{Acknowledgements}

We thank Dr. Takao Tsurui for TEM measurements and kind discussions, and Dr. Kenji Shinozaki for focused ion beam (FIB). This work was partly supported by the "High-reliability materials design and manufacturing" program at Nagaoka University of Technology. This work was partly supported by JST program, Collaborative Research Based on Industrial Demand. 


\section{References}

1 S. N. Piramanayagam: J. Appl. Phys. 102 (2007) 011301.

2 M. R. Visokay and R. Sinclair: Appl. Phys. Lett. 66 (1995) 1692.

3 T. Niizeki, Y. Utsumi, R. Aoyama, H. Yanagihara, J. Inoue, Y. Yamasaki, H. Nakao, K. Koike and E. Kita: Appl. Phys. Lett. 103 (2013) 162407.

4 A.-K. Axelsson, M. Valant, L. Fenner, A. S. Wills and N. M. Alfoed: Thin Solid Films 517 (2009) 3742. 